\title{
Tiling and spectral properties of near-cubic domains
}

\author{
by \\ Mihail N. Kolountzakis (Iraklio) and Izabella Eaba (Vancouver)
}

\begin{abstract}
We prove that if a measurable domain tiles $\mathbb{R}$ or $\mathbb{R}^{2}$ by translations, and if it is "close enough" to a line segment or a square respectively, then it admits a lattice tiling. We also prove a similar result for spectral sets in dimension 1, and give an example showing that there is no analogue of the tiling result in dimensions 3 and higher.
\end{abstract}

1. Introduction. Let $E$ be a measurable set in $\mathbb{R}^{n}$ such that $0<|E|<\infty$. We will say that $E$ tiles $\mathbb{R}^{n}$ by translations if there is a set $T \subset \mathbb{R}^{n}$ such that, up to sets of measure 0 , the sets $E+t, t \in T$, are mutually disjoint and $\bigcup_{t \in T}(E+t)=\mathbb{R}^{n}$. We call any such $T$ a translation set for $E$, and write $E+T=\mathbb{R}^{n}$. A tiling $E+T=\mathbb{R}^{n}$ is called periodic if it admits a period lattice of rank $n$; it is a lattice tiling if $T$ itself is a lattice. Here and below, a lattice in $\mathbb{R}^{n}$ will always be a set of the form $T \mathbb{Z}^{n}$, where $T$ is a linear transformation of rank $n$.

It is known ([19], [18]) that if a convex set $E$ tiles $\mathbb{R}^{n}$ by translations, it also admits a lattice tiling. A natural question is whether a similar result holds if $E$ is "sufficiently close" to being convex, e.g. if it is close enough (in an appropriate sense) to an $n$-dimensional cube. In this paper we prove that this is indeed so in dimensions 1 and 2; we also construct a counterexample in dimensions $n \geq 3$.

A major unresolved problem in the mathematical theory of tilings is the periodic tiling conjecture, which asserts that any $E$ which tiles $\mathbb{R}^{n}$ by translations must also admit a periodic tiling. (See [3] for an overview of this and other related questions.) The conjecture has been proved for all bounded measurable subsets of $\mathbb{R}([16],[12])$ and for topological discs in $\mathbb{R}^{2}$ ([2], [8]). Our Theorem 2 and Corollary 1 prove the conjecture for nearsquare domains in $\mathbb{R}^{2}$. We emphasize that no assumptions on the topology of $E$ are needed; in particular, $E$ is not required to be connected and may have infinitely many connected components.

2000 Mathematics Subject Classification: 52C20, 42A99. 
Our work was also motivated in part by a conjecture of Fuglede [1]. We call a set $E$ spectral if there is a discrete set $\Lambda \subset \mathbb{R}^{n}$, which we call a spectrum for $E$, such that $\left\{e^{2 \pi i \lambda \cdot x}: \lambda \in \Lambda\right\}$ is an orthogonal basis for $L^{2}(E)$. Fuglede conjectured that $E$ is spectral if and only if it tiles $\mathbb{R}^{n}$ by translations, and proved it under the assumption that either the translation set $T$ or the spectrum $\Lambda$ is a lattice. This problem was addressed in many recent papers (see e.g. [4], [7], [10], [13]-[17]), and in particular the conjecture has been proved for convex regions in $\mathbb{R}^{2}([9],[5],[6])$.

It follows from our Theorem 1 and from Fuglede's theorem that the conjecture is true for $E \subset \mathbb{R}$ such that $E$ is contained in an interval of length strictly less than $3|E| / 2$. (This was proved in [15] in the special case when $E$ is a union of finitely many intervals of equal length.) In dimension 2, we obtain the "tiling $\Rightarrow$ spectrum" part of the conjecture for near-square domains. Namely, if $E \subset \mathbb{R}^{2}$ tiles $\mathbb{R}^{2}$ and satisfies the assumptions of Theorem 2 or Corollary 1, it also admits a lattice tiling, hence it is a spectral set by Fuglede's theorem on the lattice case of his conjecture. We do not know how to prove the reverse implication.

Our main results are the following.

TheOREM 1. Suppose $E \subseteq[0, L]$ is measurable with measure 1 and $L=$ $3 / 2-\varepsilon$ for some $\varepsilon>0$. Let $\Lambda \subset \mathbb{R}$ be a discrete set containing 0 . Then

(a) if $E+\Lambda=\mathbb{R}$ is a tiling, it follows that $\Lambda=\mathbb{Z}$;

(b) if $\Lambda$ is a spectrum of $E$, it follows that $\Lambda=\mathbb{Z}$.

The upper bound $L<3 / 2$ in Theorem 1 is optimal: the set $[0,1 / 2] \cup$ $[1,3 / 2]$ is contained in an interval of length $3 / 2$, tiles $\mathbb{Z}$ with the translation set $\{0,1 / 2\}+2 \mathbb{Z}$, and has the spectrum $\{0,1 / 2\}+2 \mathbb{Z}$, but does not have either a lattice translation set or a lattice spectrum. This example has been known to many authors; an explicit calculation of the spectrum is given e.g. in $[14]$.

TheOREM 2. Let $E \subset \mathbb{R}^{2}$ be a measurable set such that $[0,1]^{2} \subset E \subset$ $[-\varepsilon, 1+\varepsilon]^{2}$ for $\varepsilon>0$ small enough. Assume that $E$ tiles $\mathbb{R}^{2}$ by translations. Then $E$ also admits a tiling with a lattice $\Lambda \subset \mathbb{R}^{2}$ as the translation set.

Our proof works for $\varepsilon<\varepsilon_{0} \approx 0.05496$; we do not know what is the optimal upper bound for $\varepsilon$.

Corollary 1. Let $E \subset \mathbb{R}^{2}$ be a measurable set such that $|E|=1$ and $E$ is contained in a square of sidelength $1+\varepsilon$ for $\varepsilon>0$ small enough. If $E$ tiles $\mathbb{R}^{2}$ by translations, then it also admits a lattice tiling.

Theorem 3. Let $n \geq 3$. Then for any $\varepsilon>0$ there is a set $E \subset \mathbb{R}^{n}$ with $[0,1]^{n} \subset E \subset[-\varepsilon, 1+\varepsilon]^{n}$ such that $E$ tiles $\mathbb{R}^{n}$ by translations, but does not admit a lattice tiling. 

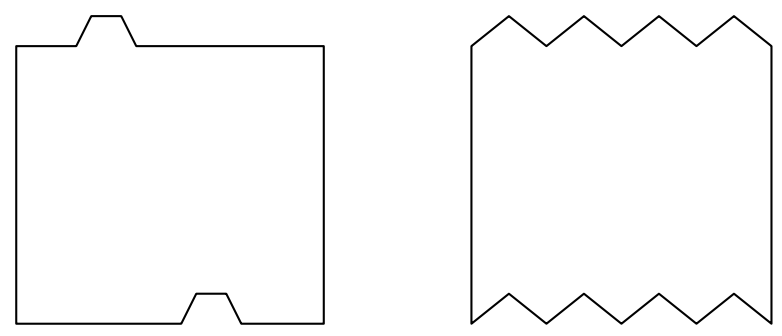

Fig. 1. Examples of near-square regions which tile $\mathbb{R}^{2}$. Note that the second region also admits aperiodic (hence non-lattice) tilings.

2. The one-dimensional case. In this section we prove Theorem 1. We shall need the following crucial lemma.

Lemma 1. Suppose that $E \subseteq[0, L]$ is measurable with measure 1 and that $L=3 / 2-\varepsilon$ for some $\varepsilon>0$. Then

$$
|E \cap(E+x)|>0 \quad \text { whenever } 0 \leq x<1 .
$$

Proof. We distinguish the cases (i) $0 \leq x \leq 1 / 2$, (ii) $1 / 2<x \leq 3 / 4$, and (iii) $3 / 4<x<1$.

(i) This is the easy case as $E \cup(E+x) \subseteq[0, L+1 / 2]=[0,2-\varepsilon]$. Since this interval has length less than 2, the sets $E$ and $E+x$ must intersect in positive measure.

(ii) Let $x=1 / 2+\alpha, 0<\alpha \leq 1 / 4$. Suppose that $|E \cap(E+x)|=0$. Then $1+2 \alpha \leq 3 / 2$ and

$$
|(E \cap[0, x]) \cup(E \cap[x, 2 x])| \leq x,
$$

as the second set does not intersect the first when shifted back by $x$. This implies that

$$
|E| \leq x+(3 / 2-\varepsilon-2 x)=3 / 2-\varepsilon-x=1-\varepsilon-\alpha<1,
$$

a contradiction as $|E|=1$. Then

(iii) Let $x=3 / 4+\alpha, 0<\alpha<1 / 4$. Suppose that $|E \cap(E+x)|=0$.

$$
|(E \cap[0,3 / 4-\alpha-\varepsilon]) \cup(E \cap[3 / 4+\alpha, 3 / 2-\varepsilon])| \leq 3 / 4-\alpha-\varepsilon,
$$

for the second set translated to the left by $x$ does not intersect the first. This implies that

$$
|E| \leq(3 / 4-\alpha-\varepsilon)+2 \alpha+\varepsilon=3 / 4+\alpha<1,
$$

a contradiction.

We need to introduce some terminology. If $f$ is a non-negative integrable function on $\mathbb{R}^{d}$ and $\Lambda$ is a subset of $\mathbb{R}^{d}$, we say that $f+\Lambda$ is a packing if, 
almost everywhere,

$$
\sum_{\lambda \in \Lambda} f(x-\lambda) \leq 1
$$

We say that $f+\Lambda$ is a tiling if equality holds almost everywhere. When $f=\chi_{E}$ is the indicator function of a measurable set, this definition coincides with the classical geometric notions of packing and tiling.

We shall need the following theorem from [10].

Theorem 4. If $f, g \geq 0, \int f(x) d x=\int g(x) d x=1$ and both $f+\Lambda$ and $g+\Lambda$ are packings of $\mathbb{R}^{d}$, then $f+\Lambda$ is a tiling if and only if $g+\Lambda$ is a tiling.

Proof of Theorem 1. (a) Suppose $E+\Lambda$ is a tiling. From Lemma 1 it follows that any two elements of $\Lambda$ differ by at least 1 . This implies that $\chi_{[0,1]}+\Lambda$ is a packing, hence it is also a tiling by Theorem 4 . Since $0 \in \Lambda$, we have $\Lambda=\mathbb{Z}$.

(b) Suppose that $\Lambda$ is a spectrum of $E$. Write

$$
\delta_{\Lambda}=\sum_{\lambda \in \Lambda} \delta_{\lambda}
$$

for the measure of one unit mass at each point of $\Lambda$. Our assumption that $\Lambda$ is a spectrum for $E$ implies that

$$
\left|\widehat{\chi}_{E}\right|^{2}+\Lambda=\mathbb{R}
$$

is a tiling (see, for example, [10]). This, in turn, implies that $\Lambda$ had density 1. Here and below, we say that a set $A \subset \mathbb{R}$ has density $\varrho$ if

$$
\lim _{N \rightarrow \infty} \frac{\#(A \cap[-N, N])}{2 N}=\varrho .
$$

Notation. The definition of the Fourier transform we use is

$$
\widehat{f}(\xi)=\int_{\mathbb{R}} e^{-2 \pi i \xi \cdot x} f(x) d x
$$

for an $L^{1}$ function $f$. If $T$ is a tempered distribution (a bounded linear functional on the Schwarz space $\mathcal{S}$ ) then its Fourier transform is defined by duality as the tempered distribution $\widehat{T}$ given by

$$
\widehat{T}(\phi)=T(\widehat{\phi}), \quad \phi \in \mathcal{S} .
$$

We now use the following result from [10]:

TheOREm 5. Suppose that $f \geq 0$ is not identically 0 , that $f \in L^{1}\left(\mathbb{R}^{d}\right)$, $\widehat{f} \geq 0$ has compact support and $\Lambda \subset \mathbb{R}^{d}$. If $f+\Lambda$ is a tiling then

$$
\operatorname{supp} \widehat{\delta}_{\Lambda} \subseteq\{\widehat{f}=0\} \cup\{0\} .
$$


Let us emphasize here that the object $\widehat{\delta}_{\Lambda}$, the Fourier transform of the tempered measure $\delta_{\Lambda}$, is in general a tempered distribution and need not be a measure.

For $f=\left|\widehat{\chi}_{E}\right|^{2}$ Theorem 5 implies

$$
\operatorname{supp} \widehat{\delta}_{\Lambda} \subseteq\{0\} \cup\left\{\chi_{E} * \widetilde{\chi}_{E}=0\right\},
$$

since $\chi_{E} * \widetilde{\chi}_{E}$ is the Fourier transform of $\left|\widehat{\chi}_{E}\right|^{2}$ (where $\widetilde{g}(x)=\overline{g(-x)}$ ). But

$$
\left\{\chi_{E} * \tilde{\chi}_{E}=0\right\}=\{x:|E \cap(E+x)|=0\} .
$$

This and Lemma 1 imply that

$$
\operatorname{supp} \widehat{\delta}_{\Lambda} \cap(-1,1)=\{0\} .
$$

Let

$$
K_{\delta}(x)=\max \{0,1-(1+\delta)|x|\}=(1+\delta) \chi_{I_{\delta}} * \widetilde{\chi}_{I_{\delta}}(x),
$$

where $I_{\delta}=[0,1 /(1+\delta)]$, be a Fejér kernel (we will later take $\delta \rightarrow 0$ ). Then

$$
\widehat{K}_{\delta}=(1+\delta)\left|\widehat{\chi}_{I_{\delta}}\right|^{2}=\frac{1+\delta}{\pi^{2} x^{2}} \sin ^{2} \frac{\pi x}{1+\delta}
$$

is a non-negative continuous function and it follows that

$$
\widehat{K}_{\delta}(0)=\frac{1}{1+\delta}
$$

and

$$
\left\{x: \widehat{K}_{\delta}(x)=0\right\}=(1+\delta)(\mathbb{Z} \backslash\{0\}) .
$$

Next, we use the following result from [11] (proved there in a more general setting):

Theorem 6. Suppose that $\Lambda \in \mathbb{R}$ is a set with density $\varrho$, that $\delta_{\Lambda}=$ $\sum_{\lambda \in \Lambda} \delta_{\lambda}$, and $\widehat{\delta}_{\Lambda}$ is a measure in a neighborhood of 0 . Then $\widehat{\delta}_{\Lambda}(\{0\})=\varrho$.

REMARK. The proof of Theorem 6 shows that the assumption of $\widehat{\delta}_{\Lambda}$ being a measure in a neighborhood of zero is superfluous, if one knows a priori that $\widehat{\delta}_{\Lambda}$ is supported only at zero, in a neighborhood of zero. Indeed, what is shown in that proof is that, as $t \rightarrow \infty$, the quantity $\widehat{\delta}_{\Lambda}(\phi(t x))$ remains bounded, for any $C_{\mathrm{c}}^{\infty}$ test function $\phi$. If $\widehat{\delta}_{\Lambda}$ were not a measure near 0 but had support only at 0 , locally, this quantity would grow like a polynomial in $t$ of degree equal to the degree of the distribution at 0 .

Applying Theorem 6 and the Remark following it we deduce that $\widehat{\delta}_{\Lambda}$ is equal to $\delta_{0}$ in a neighborhood of 0 , since $\Lambda$ has density 1 .

Next, we claim that

$$
\sum_{\lambda \in \Lambda} \widehat{K}_{\delta}(x-\lambda)=1 \quad \text { for all } x \in \mathbb{R} .
$$


Indeed, take $\psi_{\varepsilon}$ to be an even, smooth, positive-definite approximate identity, supported in $(-\varepsilon, \varepsilon)$, and take $\varepsilon=\varepsilon(\delta)$ to be small enough so that $\operatorname{supp} \psi_{\varepsilon} * K_{\delta} \subset(-1,1)$. We then have, for fixed $x$,

$$
\begin{aligned}
& \sum_{\lambda \in \Lambda} \widehat{K}_{\delta}(x-\lambda) \\
& =\lim _{\varepsilon \rightarrow 0} \sum_{\lambda \in \Lambda} \widehat{\psi}_{\varepsilon}(x-\lambda) \widehat{K}_{\delta}(x-\lambda) \\
& \left.=\lim _{\varepsilon \rightarrow 0} \delta_{\Lambda}\left(\left(\widehat{\psi}_{\varepsilon} \widehat{K}_{\delta}\right)(x-\cdot)\right) \quad \text { (by definition of } \delta_{\Lambda}\right) \\
& =\lim _{\varepsilon \rightarrow 0} \widehat{\delta}_{\Lambda}\left(e^{2 \pi i x t}\left(\psi_{\varepsilon} * K_{\delta}\right)(t)\right) \quad \text { (by definition of the FT of } \delta_{\Lambda} \text { ) } \\
& =\lim _{\varepsilon \rightarrow 0} \delta_{0}\left(e^{2 \pi i x t}\left(\psi_{\varepsilon} * K_{\delta}\right)(t)\right) \quad(\text { for } \varepsilon \text { small enough }) \\
& =\lim _{\varepsilon \rightarrow 0}\left(\psi_{\varepsilon} * K_{\delta}\right)(0)=K_{\delta}(0)=1,
\end{aligned}
$$

which establishes the claim. Applying this for $x=0$ and isolating the term $\lambda=0$ we get

$$
1=\frac{1}{1+\delta}+\sum_{0 \neq \lambda \in \Lambda} \widehat{K}_{\delta}(-\lambda) .
$$

Letting $\delta \rightarrow 0$ we obtain $\widehat{K}_{\delta}(-\lambda) \rightarrow 0$ for each $\lambda \in \Lambda \backslash\{0\}$, which implies that each such $\lambda$ is an integer, as $\mathbb{Z} \backslash\{0\}$ is the limiting set of the zeros of $\widehat{K}_{\delta}$.

To get $\Lambda=\mathbb{Z}$ notice that $\chi_{[0,1]}+\Lambda$ is a packing. By Theorem 4 we again deduce that $\chi_{[0,1]}+\Lambda$ is in fact a tiling, hence $\Lambda=\mathbb{Z}$.

\section{Planar regions}

Proof of Theorem 2. We denote the coordinates in $\mathbb{R}^{2}$ by $\left(x_{1}, x_{2}\right)$. For $0 \leq a \leq b \leq 1$ we define

$$
\begin{aligned}
& E_{1}(a, b)=\left(E \cap\left\{a \leq x_{1} \leq b, x_{2} \leq 0\right\}\right) \cup\left\{a \leq x_{1} \leq b, x_{2} \geq 0\right\}, \\
& E_{2}(a, b)=\left(E \cap\left\{a \leq x_{1} \leq b, x_{2} \geq 0\right\}\right) \cup\left\{a \leq x_{1} \leq b, x_{2} \leq 0\right\}, \\
& F_{1}(a, b)=\left(E \cap\left\{a \leq x_{2} \leq b, x_{1} \leq 0\right\}\right) \cup\left\{a \leq x_{2} \leq b, x_{1} \geq 0\right\}, \\
& F_{2}(a, b)=\left(E \cap\left\{a \leq x_{2} \leq b, x_{1} \geq 0\right\}\right) \cup\left\{a \leq x_{2} \leq b, x_{1} \leq 0\right\} .
\end{aligned}
$$

We will also use $S_{a, b}$ to denote the vertical strip $[a, b] \times \mathbb{R}$. Let $v=\left(v_{1}, v_{2}\right) \in \mathbb{R}^{2}$. We will say that $E_{2}(a, b)$ complements $E_{1}\left(a^{\prime}, b^{\prime}\right)+v$ if $E_{1}\left(a^{\prime}, b^{\prime}\right)+v$ is positioned above $E_{2}(a, b)$ so that (up to sets of measure 0 ) the two sets are disjoint and their union is $S_{a, b}$. In particular, we must have $a^{\prime}+v_{1}=a$ and $b^{\prime}+v_{1}=b$. We will also say that $F_{2}(a, b)$ complements $F_{1}\left(a^{\prime}, b^{\prime}\right)+v$ if the obvious analogue of the above statement holds. We will write $\widetilde{E}_{1}(a, b)=$ 
$S_{a, b} \backslash E_{1}(a, b)$, and similarly for $E_{2}$. Finally, we write $A \sim B$ if the sets $A$ and $B$ are equal up to sets of measure 0 .

Lemma 2. Let $0<s^{\prime \prime}<s^{\prime}<s<2 s^{\prime \prime}$. Suppose that $E_{1}(a, a+s)+v$, $E_{1}\left(a, a+s^{\prime}\right)+v^{\prime}, E_{1}\left(a, a+s^{\prime \prime}\right)+v^{\prime \prime}$ complement $E_{2}(b-s, b), E_{2}\left(b-s^{\prime}, b\right)$, $E_{2}\left(b-s^{\prime \prime}, b\right)$ respectively. Then the points $v, v^{\prime}, v^{\prime \prime}$ are collinear. Moreover, the absolute value of the slope of the line through $v, v^{\prime \prime}$ is bounded by $\varepsilon\left(2 s^{\prime \prime}-s\right)^{-1}$.

Applying the lemma to the symmetric reflection of $E$ about the line $x_{2}=1 / 2$, we find that the conclusions of the lemma also hold if we assume that $E_{2}(a, a+s)+v, E_{2}\left(a, a+s^{\prime}\right)+v^{\prime}, E_{2}\left(a, a+s^{\prime \prime}\right)+v^{\prime \prime}$ complement $E_{1}(b-s, b), E_{1}\left(b-s^{\prime}, b\right), E_{1}\left(b-s^{\prime \prime}, b\right)$ respectively. Furthermore, we may interchange the $x_{1}$ and $x_{2}$ coordinates and obtain the analogue of the lemma with $E_{1}, E_{2}$ replaced by $F_{1}, F_{2}$.

Proof of Lemma 2. Let $v=\left(v_{1}, v_{2}\right), v^{\prime}=\left(v_{1}^{\prime}, v_{2}^{\prime}\right), v^{\prime \prime}=\left(v_{1}^{\prime \prime}, v_{2}^{\prime \prime}\right)$. We first observe that if $v_{1}=v_{1}^{\prime \prime}$, it follows from the assumptions that $v=v^{\prime \prime}$ and there is nothing to prove. We may therefore assume that $v_{1} \neq v_{1}^{\prime \prime}$. We do, however, allow $v^{\prime}=v$ or $v^{\prime}=v^{\prime \prime}$.

It follows from the assumptions that $E_{2}\left(b-s^{\prime \prime}, b\right)$ complements each of $E_{1}\left(a, a+s^{\prime \prime}\right)+v^{\prime \prime}, E_{1}\left(a+s^{\prime}-s^{\prime \prime}, a+s^{\prime}\right)+v^{\prime}, E_{1}\left(a+s-s^{\prime \prime}, a+s\right)+v$. Hence

$$
\begin{aligned}
E_{1}\left(a+s^{\prime}-s^{\prime \prime}, a+s^{\prime}\right) & \sim E_{1}\left(a, a+s^{\prime \prime}\right)+\left(v^{\prime \prime}-v^{\prime}\right), \\
E_{1}\left(a+s-s^{\prime \prime}, a+s\right) & \sim E_{1}\left(a, a+s^{\prime \prime}\right)+\left(v^{\prime \prime}-v\right) .
\end{aligned}
$$

Let $n$ be the unit vector perpendicular to $v-v^{\prime \prime}$ and such that $n_{2}>0$. For $t \in \mathbb{R}$, let $P_{t}=\{x: x \cdot n \leq t\}$. We define for $0 \leq c \leq c^{\prime} \leq 1$ :

$$
\begin{aligned}
& \alpha_{c, c^{\prime}}=\inf \left\{t \in \mathbb{R}:\left|E_{1}\left(c, c^{\prime}\right) \cap P_{t}\right|>0\right\}, \\
& \beta_{c, c^{\prime}}=\sup \left\{t \in \mathbb{R}:\left|\widetilde{E}_{1}\left(c, c^{\prime}\right) \backslash P_{t}\right|>0\right\} .
\end{aligned}
$$

We will say that $x$ is a low point of $E_{1}\left(c, c^{\prime}\right)$ if $x \in S_{c, c^{\prime}}, x \cdot n=\alpha_{c, c^{\prime}}$, and for any open $\operatorname{disc} D$ centered at $x$ we have

$$
\left|D \cap E_{1}\left(c, c^{\prime}\right)\right|>0 .
$$

Similarly, we call $y$ a high point of $\widetilde{E}_{1}\left(c, c^{\prime}\right)$ if $y \in S_{c, c^{\prime}}, y \cdot n=\beta_{c, c^{\prime}}$, and for any open $\operatorname{disc} D$ centered at $y$ we have

$$
\left|D \cap \widetilde{E}_{1}\left(c, c^{\prime}\right)\right|>0 .
$$

It is easy to see that such points $x, y$ actually exist. Indeed, by the definition of $\alpha_{c, c^{\prime}}$ and an obvious covering argument, for any $\alpha>\alpha_{c, c^{\prime}}$ there are points $x^{\prime}$ such that $x^{\prime} \cdot n \leq \alpha$ and that (6) holds for any disc $D$ centered at $x^{\prime}$. Thus the set of such points $x^{\prime}$ has at least one accumulation point $x$ on the line $x \cdot n=\alpha_{c, c^{\prime}}$. It follows that any such $x$ is a low point of $E_{1}\left(c, c^{\prime}\right)$. The same argument works for $y$. 
The low and high points need not be unique; however, all low points $x$ of $E_{1}\left(c, c^{\prime}\right)$ lie on the same line $x \cdot n=\alpha_{c, c^{\prime}}$ parallel to the vector $v-v^{\prime \prime}$, and similarly for high points. Furthermore, the low and high points of $E_{1}\left(c, c^{\prime}\right)$ do not change if $E_{1}\left(c, c^{\prime}\right)$ is modified by a set of measure 0 .

Let now $A=E_{1}\left(a, a+s^{\prime \prime}\right)$, and let $x$ be a low point of $A$. Since $s<2 s^{\prime \prime}$, we have

$B:=E_{1}(a, a+s)=E_{1}\left(a, a+s^{\prime \prime}\right) \cup E_{1}\left(a+s-s^{\prime \prime}, a+s\right) \sim A \cup\left(A+v^{\prime \prime}-v\right)$, hence $x$ is also a low point of $B$ with respect to $v-v^{\prime \prime}$. Now note that

$$
E_{1}\left(a+s^{\prime}-s^{\prime \prime}, a+s^{\prime}\right) \sim A+\left(v^{\prime \prime}-v^{\prime}\right)
$$

intersects any open neighborhood of $x+\left(v^{\prime \prime}-v^{\prime}\right)$ in positive measure. But on the other hand, $E_{1}\left(a+s^{\prime}-s^{\prime \prime}, a+s^{\prime}\right) \subset B$. By the extremality of $x$ in $B$, $x+\left(v^{\prime \prime}-v^{\prime}\right)$ lies on or above the line segment joining $x$ and $x+\left(v^{\prime \prime}-v\right)$, hence $v^{\prime \prime}-v^{\prime}$ lies on or above the line segment joining 0 and $v^{\prime \prime}-v$.

Repeating the argument in the last paragraph with $x$ replaced by a high point $y$ of $\widetilde{E}_{1}\left(a, a+s^{\prime \prime}\right)$, we deduce that $v^{\prime \prime}-v^{\prime}$ lies on or below the line segment joining 0 and $v^{\prime \prime}-v$. Hence $v, v^{\prime}, v^{\prime \prime}$ are collinear.

Finally, we estimate the slope of the line through $v, v^{\prime \prime}$. We have to prove that

$$
\frac{2 s^{\prime \prime}-s}{s-s^{\prime \prime}}\left|v_{2}^{\prime \prime}-v_{2}\right| \leq \varepsilon
$$

(recall that $v_{1}^{\prime \prime}-v_{1}=s-s^{\prime \prime}$ ). Define $x$ as above, and let $k \in \mathbb{Z}$. Iterating translations by $v-v^{\prime \prime}$ (in both directions), we find that $x+k\left(v-v^{\prime \prime}\right)$ is a low point of $B$ as long as it belongs to $B$, i.e. as long as

$$
a \leq x_{1}+k\left(s-s^{\prime \prime}\right) \leq a+s .
$$

The number of such $k$ 's is at least $s /\left(s-s^{\prime \prime}\right)-1$. On the other hand, all low points of $B$ lie in the rectangle $a \leq x_{1} \leq a+s,-\varepsilon \leq x_{2} \leq 0$. Hence

$$
\left(\frac{s}{s-s^{\prime \prime}}-2\right)\left|v_{2}^{\prime \prime}-v_{2}\right| \leq \varepsilon
$$

which is (8).

We return to the proof of Theorem 2. Since $E$ is almost a square, we know roughly how the translates of $E$ can fit together. Locally, any tiling by $E$ is essentially a tiling by a "solid" $1 \times 1$ square with "margins" of width between 0 and $2 \varepsilon$ (see Fig. 2).

We first locate a "corner". Namely, we may assume that the tiling contains $E$ and its translates $E+u, E+v$, where

$$
\begin{aligned}
& 1 \leq u_{1} \leq 1+2 \varepsilon, \quad-2 \varepsilon \leq u_{2} \leq 2 \varepsilon, \\
& 0 \leq v_{1} \leq \frac{1}{2}+\varepsilon, \quad 1 \leq v_{2} \leq 1+2 \varepsilon .
\end{aligned}
$$




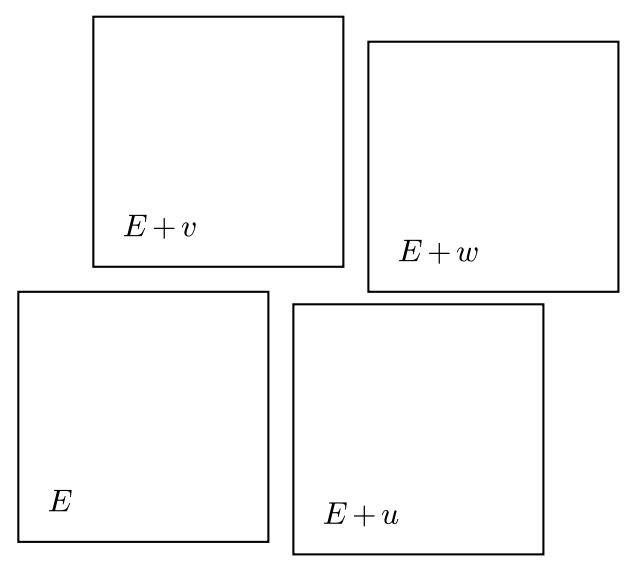

Fig. 2. A "corner" and a fourth near-square

This can always be achieved by translating the tiled plane and taking symmetric reflections of it if necessary.

Let $E+w$ be the translate of $E$ which fits into this corner:

$$
v_{1}+1 \leq w_{1} \leq v_{1}+1+2 \varepsilon, \quad u_{2}+1 \leq w_{2} \leq u_{2}+1+2 \varepsilon .
$$

We will prove that $w=u+v$ (without the $\varepsilon$-errors).

From (11), (9), (10) we have

$$
1 \leq w_{1} \leq \frac{3}{2}+3 \varepsilon, \quad-4 \varepsilon \leq w_{2}-v_{2} \leq 4 \varepsilon .
$$

Observe also that any points $\left(x_{1}, x_{2}\right)$ between $E+u$ and $E+w$ that belong to tiles other than $E+u$ or $E+w$ must have $x_{1} \leq w+\varepsilon$ or $x_{1} \geq u+1-\varepsilon$, since otherwise the solid square belonging to the same tile would overlap at least one of the solid squares belonging to $E+u$ or $E+w$. A similar statement holds for $E+v$ and $E+w$. Hence $w$ satisfies both of the following. and

(A) $E_{1}\left(\varepsilon, 1-\left(w_{1}-u_{1}\right)-\varepsilon\right)$ complements $E_{2}\left(w_{1}-u_{1}+\varepsilon, 1-\varepsilon\right)+(u-w)$,

$$
\begin{array}{r}
1-\left(w_{1}-u_{1}\right)-2 \varepsilon \geq 1+1-\left(\frac{3}{2}+3 \varepsilon\right)-2 \varepsilon=\frac{1}{2}-5 \varepsilon, \quad\left|w_{1}-v_{1}-1\right| \leq 2 \varepsilon . \\
\text { (B) }-4 \varepsilon \leq w_{2}-v_{2} \leq 4 \varepsilon, u_{2}+1 \leq w_{2} \leq u_{2}+1+2 \varepsilon, \text { and } F_{2}(r, t)
\end{array}
$$
complements $F_{1}(\widetilde{r}, \widetilde{t})+(w-v)$, where

$$
\begin{array}{ll}
r=\max \left(0, w_{2}-v_{2}\right)+\varepsilon, & \widetilde{r}=\max \left(0, v_{2}-w_{2}\right)+\varepsilon, \\
t=1-\max \left(0, v_{2}-w_{2}\right)-\varepsilon, & \widetilde{t}=1-\max \left(0, w_{2}-v_{2}\right)-\varepsilon .
\end{array}
$$

If $w=u+v$, we have $w-u=v, w-v=u$, hence by considering the "corner" $E, E+u, E+v$ we see that both (A) and (B) hold. Assuming that $\varepsilon$ is small enough, we shall prove that: 
$1^{\circ}$ All points $w$ satisfying (A) lie on a fixed straight line $l_{1}$ with slope $m_{1}$, where $\left|m_{1}\right| \leq \varepsilon(1 / 2-9 \varepsilon)^{-1}$.

$2^{\circ}$ All points $w$ satisfying (B) lie on a fixed straight line $l_{2}$ with slope $m_{2}$, where $\left|m_{2}\right| \geq \varepsilon^{-1}(1-8 \varepsilon)$.

If $\varepsilon<(13-3 \sqrt{3}) / 142 \approx 0.05496$ (the smaller root of the equation $\left.71 \varepsilon^{2}-13 \varepsilon+1 / 2=0\right)$, the upper bound for $\left|m_{1}\right|$ is less than the lower bound for $\left|m_{2}\right|$. It follows that there can be at most one $w$ which satisfies both (A) and (B), since $l_{1}$ and $l_{2}$ intersect only at one point. Consequently, if $E+w$ is the translate of $E$ chosen as above, we must have $w=u+v$. Now it is easy to see that $E+\Lambda$ is a tiling, where $\Lambda$ is the lattice $\{k u+m v: k, m \in \mathbb{Z}\}$.

We first prove $1^{\circ}$. Suppose that $w, w^{\prime}, w^{\prime \prime}, \ldots$ (not necessarily all distinct) satisfy (A). By the assumptions in (A), we may apply Lemma 2 with $E_{1}$ and $E_{2}$ interchanged and with $a=0, b=1, s=1-\left(w_{1}-u_{1}\right), s^{\prime}=$ $1-\left(w_{1}^{\prime}-u_{1}\right), \ldots \geq 1 / 2-5 \varepsilon$. From the second inequality in (A) and the triangle inequality we also have $\left|s-s^{\prime \prime}\right| \leq 4 \varepsilon$. We find that all $w$ satisfying (A) lie on a line $l_{1}$ with slope bounded by

$$
\frac{\varepsilon}{\left|2 s^{\prime \prime}-s\right|} \leq \frac{\varepsilon}{s^{\prime \prime}-\left|s^{\prime \prime}-s\right|} \leq \frac{\varepsilon}{1 / 2-9 \varepsilon} \text {. }
$$

To prove $2^{\circ}$, we let $w, w^{\prime}, w^{\prime \prime}$ be three (not necessarily distinct) points satisfying (B) and such that $w_{2} \leq w_{2}^{\prime} \leq w_{2}^{\prime \prime}$. Observe that $r \leq r^{\prime} \leq r^{\prime \prime}$ and $t \geq t^{\prime} \geq t^{\prime \prime}$ (the notation is self-explanatory). We then apply the obvious analogue of Lemma 2 with $E_{1}, E_{2}$ replaced by $F_{1}, F_{2}$ and with $a=r^{\prime \prime}$, $s=t-r^{\prime \prime}, s^{\prime}=t^{\prime}-r^{\prime \prime}, s^{\prime \prime}=t^{\prime \prime}-r^{\prime \prime}, b=\widetilde{t}^{\prime \prime}$. From the estimates in (B) we have

$$
\begin{gathered}
\left|s-s^{\prime \prime}\right|=\left|t-t^{\prime \prime}\right| \leq\left|w_{2}-w_{2}^{\prime \prime}\right| \leq 2 \varepsilon, \\
s^{\prime \prime}=t^{\prime \prime}-r^{\prime \prime}=1-\max \left(0, v_{2}-w_{2}^{\prime \prime}\right)-\max \left(0, w_{2}^{\prime \prime}-v_{2}\right)-2 \varepsilon \geq 1-6 \varepsilon,
\end{gathered}
$$

hence $\left|2 s^{\prime \prime}-s\right| \geq s^{\prime \prime}-\left|s-s^{\prime \prime}\right| \geq 1-8 \varepsilon$. We conclude that all $w$ satisfying (B) lie on a line $l_{2}$ such that the inverse of the absolute value of its slope is bounded by $\varepsilon /(1-8 \varepsilon)$.

Proof of Corollary 1. Let $Q=[0,1] \times[0,1]$. By rescaling, it suffices to prove that for any $\varepsilon>0$ there is a $\delta>0$ such that if $E \subset Q, E$ tiles $\mathbb{R}^{2}$ by translations, and $|E| \geq 1-\delta$, then $E$ contains the square

$$
Q_{\varepsilon}=[\varepsilon, 1-\varepsilon] \times[\varepsilon, 1-\varepsilon]
$$

(up to sets of measure 0 ). The result then follows from Theorem 2.

Let $E$ be as above, and suppose that $Q_{\varepsilon} \backslash E$ has positive measure. Since $E$ tiles $\mathbb{R}^{2}$, there is a $v \in \mathbb{R}^{2}$ such that $|E \cap(E+v)|=0$ and $\left|Q_{\varepsilon} \cap(E+v)\right|>0$. We then have

$$
|E \cup(E+v)|=|E|+|E+v| \geq 2-2 \delta,
$$


but also

$$
|E \cup(E+v)| \leq|Q \cup(Q+v)| \leq 2-\varepsilon^{2},
$$

since $E \subset Q, E+v \subset Q+v$, and $Q_{\varepsilon} \cap(Q+v) \neq \emptyset$ so that $|Q \cap(Q+v)| \geq \varepsilon^{2}$. This is a contradiction if $\delta$ is small enough.

4. A counterexample in higher dimensions. In this section we prove Theorem 3 . It suffices to construct $E$ for $n=3$, since then $E \times[0,1]^{n-3}$ is a subset of $\mathbb{R}^{n}$ with the required properties.

Let $\left(x_{1}, x_{2}, x_{3}\right)$ denote the Cartesian coordinates in $\mathbb{R}^{3}$. It will be convenient to rescale $E$ so that $[\varepsilon, 1]^{3} \subset E \subset[0,1+\varepsilon]^{3}$.

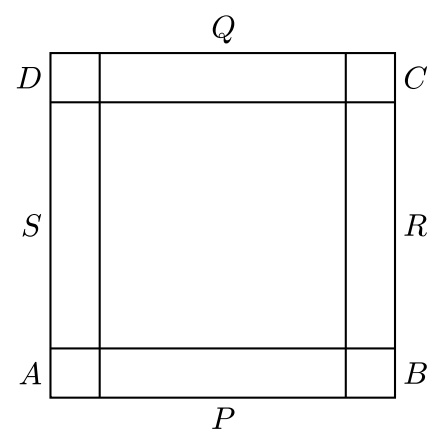

Fig. 3. The construction of $E$

We construct $E$ as follows. We let $E$ be bounded from below and above by the planes $x_{3}=0$ and $x_{3}=1$ respectively. The planes $x_{1}=\varepsilon, x_{1}=1$, $x_{2}=\varepsilon, x_{2}=1$ divide the cube $[0,1+\varepsilon]^{3}$ into 9 parts (Figure 3 ). The middle part is entirely contained in $E$. We label by $A, B, C, D, P, Q, R, S$ the remaining 8 segments as shown in Figure 3. We then let

$$
\begin{aligned}
& E \cap P=P \cap\left\{0 \leq x_{3} \leq 1 / 8 \text { or } 1 / 2 \leq x_{3} \leq 5 / 8\right\} \\
& E \cap R=R \cap\left\{0 \leq x_{3} \leq 1 / 8 \text { or } 1 / 2 \leq x_{3} \leq 5 / 8\right\} \\
& E \cap Q=Q \cap\left\{0 \leq x_{3} \leq 1 / 4 \text { or } 3 / 8 \leq x_{3} \leq 3 / 4 \text { or } 7 / 8 \leq x_{3} \leq 1\right\} \\
& E \cap S=S \cap\left\{0 \leq x_{3} \leq 1 / 4 \text { or } 3 / 8 \leq x_{3} \leq 3 / 4 \text { or } 7 / 8 \leq x_{3} \leq 1\right\} \\
& E \cap A=A \cap\left\{0 \leq x_{3} \leq 1 / 16\right\} \\
& E \cap C=A \cap\left\{1 / 2 \leq x_{3} \leq 9 / 16\right\} \\
& E \cap B=B \cap\left\{5 / 16 \leq x_{3} \leq 3 / 4\right\} \\
& E \cap D=D \cap\left\{0 \leq x_{3} \leq 1 / 4 \text { or } 13 / 16 \leq x_{3} \leq 1\right\} .
\end{aligned}
$$

We also define $K=\bigcup_{j \in \mathbb{Z}}(E+(0,0, j))$.

Let $E+T$ be a tiling of $\mathbb{R}^{3}$, and assume that $0 \in T$. Suppose that $E+v$ and $E+w$ are neighbors in this tiling so that the vertical sides of $(E \cap P)+v$ and $(E \cap Q)+w$ meet in a set of non-zero two-dimensional measure. Then 
we must have $v-w=\left(0,1,(v-w)_{3}\right)$, where $(v-w)_{3} \in\{ \pm 1 / 4, \pm 3 / 4\}$. A similar statement holds with $P, Q$ replaced by $R, S$ and with the $x_{1}, x_{2}$ coordinates interchanged. We deduce that the tiling consists of copies of $E$ stacked into identical vertical "columns" $K_{i j}=K+\left(i, j, t_{i j}\right)$, arranged in a rectangular grid in the $x_{1} x_{2}$ plane and shifted vertically so that $t_{i+1, j}-t_{i j}$ and $t_{i, j+1}-t_{i j}$ are always $\pm 1 / 4$. We will use matrices $\left(t_{i j}\right)$ to encode such a tiling or portions thereof.

It is easy to see that $\left(t_{i j}\right)$, where $t_{i j}=0$ if $i+j$ is even and $1 / 4$ if $i+j$ is odd, is indeed a tiling. It remains to show that $E$ does not admit a lattice tiling. Indeed, the four possible choices of the generating vectors in any lattice $\left(t_{i j}\right)$ with $t_{i j}= \pm 1 / 4$ produce the configurations

$$
\left(\begin{array}{cc}
0 & t \\
t & 2 t
\end{array}\right), \quad\left(\begin{array}{cc}
2 t & t \\
t & 0
\end{array}\right), \quad\left(\begin{array}{cc}
0 & t \\
-t & 0
\end{array}\right), \quad\left(\begin{array}{cc}
0 & -t \\
t & 0
\end{array}\right) .
$$

But it is easy to see that the corners $A, B, C, D$ do not match if so translated.

\section{References}

[1] B. Fuglede, Commuting self-adjoint partial differential operators and a group-theoretic problem, J. Funct. Anal. 16 (1974), 101-121.

[2] D. Girault-Beauquier and M. Nivat, Tiling the plane with one tile, in: Topology and Category Theory in Computer Science, G. M. Reed, A. W. Roscoe and R. F. Wachter (eds.), Oxford Univ. Press, 1989, 291-333.

[3] B. Grünbaum and G. C. Shephard, Tilings and Patterns, Freeman, New York, 1987.

[4] A. Iosevich, N. H. Katz and S. Pedersen, Fourier bases and a distance problem of Erdös, Math. Res. Lett. 6 (1999), 251-255.

[5] A. Iosevich, N. H. Katz and T. Tao, Convex bodies with a point of curvature do not have Fourier bases, Amer. J. Math. 123 (2001), 115-120.

[6] - - - - Fuglede conjecture holds for convex planar domains, Math. Res. Lett., to appear.

[7] P. Jorgensen and S. Pedersen, Spectral pairs in Cartesian coordinates, J. Fourier Anal. Appl. 5 (1999), 285-302.

[8] R. Kenyon, Rigidity of planar tilings, Invent. Math. 107 (1992), 637-651.

[9] M. N. Kolountzakis, Non-symmetric convex domains have no basis of exponentials, Illinois J. Math. 44 (2000), 542-550.

[10] -, Packing, tiling, orthogonality and completeness, Bull. London Math. Soc. 32 (2000), 589-599.

[11] - On the structure of multiple translational tilings by polygonal regions, Discrete Comput. Geom. 23 (2000), 537-553.

[12] M. N. Kolountzakis and J. C. Lagarias, Structure of tilings of the line by a function, Duke Math. J. 82 (1996), 653-678.

[13] M. N. Kolountzakis and M. Papadimitrakis, A class of non-convex polytopes that admit no orthonormal basis of exponentials, Illinois J. Math. 46 (2002), 1227-1232.

[14] I. Łaba, Fuglede's conjecture for a union of two intervals, Proc. Amer. Math. Soc. 121 (2001), 2965-2972.

[15] - , The spectral set conjecture and multiplicative properties of roots of polynomials, J. London Math. Soc. (2) 65 (2002), 661-671. 
[16] J. Lagarias and Y. Wang, Tiling the line with translates of one tile, Invent. Math. 124 (1996), 341-365.

[17] - - - Spectral sets and factorization of finite abelian groups, J. Funct. Anal. 73 (1997), 122-134.

[18] P. McMullen, Convex bodies which tile the space by translation, Mathematika 27 (1980), 113-121.

[19] B. A. Venkov, On a class of Euclidean polyhedra, Vestnik Leningrad. Univ. Ser. Mat. Fiz. Khim. 9 (1954), no. 2, 11-31 (in Russian).

Department of Mathematics

Department of Mathematics University of Crete

Knossos Ave.

71409 Iraklio, Greece University of British Columbia

E-mail: mk@fourier.math.uoc.gr Vancouver, B.C. V6T 1Z2, Canada

E-mail: ilaba@math.ubc.ca

Received October 15, 2002

Revised version June 17, 2003 